\title{
ARTICLE
}

\section{Interplay between Alzheimer's disease and global glucose metabolism revealed by the metabolic profile alterations of pancreatic tissue and serum in APP/PS1 transgenic mice}

\author{
Xia Liu' ${ }^{1}$, Wei Wang ${ }^{2,3}$, Hua-li Chen ${ }^{1}$, Hai-yan Zhang ${ }^{2,3,4}$ and Nai-xia Zhang ${ }^{1,4}$
}

\begin{abstract}
Increasing evidence suggests that there is a correlation between type 2 diabetes mellitus (T2D) and Alzheimer's disease (AD). Increased $A \beta$ polypeptide production in $A D$ patients would promote metabolic abnormalities, insulin signaling dysfunction and perturbations in glucose utilization, thus leading to the onset of T2D. However, the metabolic mechanisms underlying the interplay between $A D$ and its diabetes-promoting effects are not fully elucidated. Particularly, systematic metabolomics analysis has not been performed for the pancreas tissues of AD subjects, which play key roles in the glucose metabolism of living systems. In the current study, we characterized the dynamic metabolic profile alterations of the serum and the pancreas of APP/PS1 double-transgenic mice (an AD mouse model) using the untargeted metabolomics approaches. Serum and pancreatic tissues of APP/PS1 transgenic mice and wild-type mice were extracted and subjected to NMR analysis to evaluate the functional state of pancreas in the progress of AD. Multivariate analysis of principal component analysis (PCA) and orthogonal partial least squares discriminant analysis (OPLS-DA) were conducted to define the global and the local (pancreas) metabolic features associated with the possible initiation of $T 2 D$ in the progress of $A D$. Our results showed the onset of AD-induced global glucose metabolism disorders in $A D$ mice. Hyperglycemia and its accompanying metabolic disorders including energy metabolism down-regulation and oxidative stress were observed in the serum of AD mice. Meanwhile, global disturbance of branched-chain amino acid (BCAA) metabolism was detected, and the change of BCAA (leucine) was positively correlated to the alteration of glucose. Moreover, increased level of glucose and enhanced energy metabolism were observed in the pancreas of AD mice. The results suggest that the diabetes-promoting effects accompanying the progress of $A D$ are achieved by down-regulating the global utilization of glucose and interfering with the metabolic function of pancreas. Since T2D is a risk factor for the pathogenesis of AD, our findings suggest that targeting the glucose metabolism dysfunctions might serve as a supplementary therapeutic strategy for Alzheimer's disease.
\end{abstract}

Keywords: Alzheimer's disease; type 2 diabetes; APP/PS1 transgenic mice; metabolomics; NMR; glucose metabolism dysfunctions

Acta Pharmacologica Sinica (2019) 40:1259-1268; https://doi.org/10.1038/s41401-019-0239-3

\section{INTRODUCTION}

Over the past decades, rapidly increasing evidence has indicated that there is a correlation between type 2 diabetes (T2D) mellitus and Alzheimer's disease (AD) [1-3]. Metabolic abnormalities, insulin signaling dysfunction and perturbations in glucose utilization are the common backgrounds shared by the disease pathogenesis of both $A D$ and T2D $[4,5]$. One of the prominent pathological hallmarks of $A D$ is the amyloid plaques formed within the brain by the aggregation of $\beta$-amyloid $(A \beta)$ polypeptides. However, the remarkable $A \beta$ polypeptide deposition is not only restrained to the brains of $A D$ subjects; accumulation of $A \beta$ polypeptides within the pancreatic islets of $A D$ mice $[4,6-8]$ and diabetes patients [9] has also been reported. Both insulin and $A \beta$ polypeptides are substrates of insulin-degrading enzyme (IDE) [10]. The accumulation of amylin in the progression of $A D$ has been demonstrated to deplete the activity of IDE and then induce hyperinsulinemia and insulin resistance in $A D$ mice $[11,12]$. In addition to the amyloid plaques formed by $A \beta$ polypeptides, the soluble forms of $A \beta$ peptides also play a role in $A D$-induced insulin signaling disorder by binding to the insulin receptor in the brain $[13,14]$. All of these findings described above spur enthusiasm for the systematic investigation of the underlying metabolic mechanisms accompanying the pathogenesis of Alzheimer's disease and its complication, type 2 diabetes $[2,15]$. However, although efforts have been made, the metabolic interplay between Alzheimer's disease and glucose metabolism dysfunctions has not yet been fully elucidated. To the best of our knowledge, systematic metabolomics analysis has never been conducted on pancreatic tissues of $A D$ subjects, which play key roles in the glucose metabolism of living systems.

In this manuscript, to reveal the metabolic interplay between Alzheimer's disease and global glucose metabolism in a spatial and

\footnotetext{
${ }^{1}$ Department of Analytical Chemistry, Shanghai Institute of Materia Medica, Chinese Academy of Sciences, Shanghai 201203 , China; ${ }^{2}$ State Key Laboratory of Drug Research,

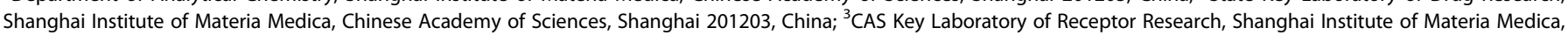
Chinese Academy of Sciences, Shanghai 201203, China and ${ }^{4}$ University of the Chinese Academy of Sciences, Beijing 100049, China

Correspondence: Hai-yan Zhang (hzhang@simm.ac.cn) or Nai-xia Zhang (nxzhang@simm.ac.cn)

These authors contributed equally: Xia Liu, Wei Wang
}

Received: 16 December 2018 Accepted: 11 April 2019

Published online: 14 May 2019 
temporal context, we performed a nuclear magnetic resonance (NMR)-based metabolomics study using an AD mouse model (APP/ PS1 transgenic mice). The serum and pancreatic tissues of APP/PS1 transgenic mice and wild-type mice were extracted and subjected to NMR analysis to evaluate the functional state of the pancreas in the progression of Alzheimer's disease. Multivariate analysis of principal component analysis (PCA) and orthogonal partial least squares discriminant analysis (OPLS-DA) were conducted to define the global and local (pancreas) metabolic features associated with the possible initiation of T2D in the progress of AD. The results achieved through this study provide new insights into the underlying molecular mechanisms for understanding the interplay between $\mathrm{AD}$ and T2D-favored metabolic disorders. In addition, the hypothesis that $A D$ is a heterogeneous disease with both central and peripheral manifestations is supported by the metabolomics data presented in this manuscript, which suggests that targeting the peripheral manifestations of $A D$ might serve as an alternative strategy to effectively treat this disease, which currently has no definitive cure.

\section{MATERIALS AND METHODS}

Materials

Sodium chloride, $\mathrm{NaN}_{3}, \mathrm{NaH}_{2} \mathrm{PO}_{4} \cdot 2 \mathrm{H}_{2} \mathrm{O}, \mathrm{Na}_{2} \mathrm{HPO}_{4} \cdot 12 \mathrm{H}_{2} \mathrm{O}$ (all in analytical grade), $\mathrm{CHCl}_{3}$, and $\mathrm{CH}_{3} \mathrm{OH}$ (HPLC grade) were purchased from Sinopharm Chemical Reagent Co. Ltd. (Shanghai, China). $\mathrm{D}_{2} \mathrm{O}$ (99.9\% in D) containing sodium 3-(trimethyl-silyl) propionate-2, 2, 3, 3 , d4 (TSP), which was used as an internal chemical shift reference standard, was provided by Sigma-Aldrich (Sigma Chemical Corp., St. Louis, MO, USA). To prevent $\mathrm{pH}$ from affecting the chemical shift at different concentrations, a buffer system containing $0.2 \% \mathrm{NaN}_{3}$ and $0.2 \mathrm{M} \mathrm{Na}_{2} \mathrm{HPO}_{4} / \mathrm{NaH}_{2} \mathrm{PO}_{4}$ dissolved in $\mathrm{D}_{2} \mathrm{O}$ at $\mathrm{pH} 7.4$ was prepared and used for NMR sample preparation.

\section{Animals}

A well-characterized $A D$ mouse model coexpressing human amyloid precursor protein (APP695) with the Swedish K670M/ $671 \mathrm{NL}$ mutation and human presenilin 1 with the exon-9 deletion mutation (PSEN1dE9), abbreviated as the APP/PS1 mouse model, was chosen and used in the present study. The genetic background of the APP/PS1 mice used in this study was C57BL/ 6. APP/PS1 mice at the ages of 6 month (APP $6 \mathrm{~m}, n=9$, male/ female: $3 / 6$ ) and 9 month (APP9m, $n=10$, male/female: $5 / 5$ ) and their wild-type (WT) littermates (C57BL/6) (WT6m, $n=9$, male/ female: $4 / 5$; WT9m, $n=10$, male/female: $3 / 7$ ) were obtained from the Laboratory Animal Center, Shanghai Institute of Materia Medica, Chinese Academy of Sciences (Shanghai, China). All experimental procedures used in this study followed the National Institutes of Health Guide for the Care and Use of Laboratory Animals, as well as the guidelines of the Animal Care and Use Committee of Shanghai Institute of Materia Medica. The animals were housed under a $12 \mathrm{~h} / 12 \mathrm{~h}$ light/dark cycle with free access to food and water for 1 week before biosample collection.

Serum and pancreas extraction procedures

Blood samples were collected in marked pro-coagulation tubes from the retinal vein plexus of experimental mice and then centrifuged $\left(5000 \times g, 10 \mathrm{~min}, 4^{\circ} \mathrm{C}\right)$ to remove any precipitates. The supernatants of serum samples were aliquoted in Eppendorf tubes and frozen at $-80^{\circ} \mathrm{C}$ until NMR data acquisition. The whole pancreatic tissues were quickly removed after the experimental mice were killed by dislocation of the cervical vertebrae, immediately snap-frozen in liquid nitrogen and then stored at $-80^{\circ} \mathrm{C}$ until further analysis. Extractions of polar metabolites from the pancreas tissues were performed following the reported procedure [16-18]. In short, tissue samples, which were preweighed and thawed on ice, were homogenized and extracted with an ice-cold methanol-chloroform-water solvent system (4, 4, and $2.85 \mu \mathrm{L}$, respectively per $\mathrm{mg} /$ tissue). The mixtures were allowed to thaw for $3 \mathrm{~min}$ and then homogenized by the $2 \times$ $20 \mathrm{~s}$ beating of $5600 \mathrm{r} / \mathrm{min}$ with a $20 \mathrm{~s}$ pause between the bead beatings using a tissue homogenizer (Precellys 24, Bertin Technologies, Villeurbanne, France). After the samples were bathed in ice for $15 \mathrm{~min}$, they were centrifuged at $12000 \times \mathrm{g}$ at $4{ }^{\circ} \mathrm{C}$ for $20 \mathrm{~min}$, forming two phases, with protein and cellular debris as the intermediate layer. The methanol/water upper phase (containing polar metabolites) was lyophilized and reconstituted in $600 \mu \mathrm{L}$ NMR buffer containing $550 \mu \mathrm{L}$ phosphate buffer $(0.2 \mathrm{M}$ $\mathrm{Na}_{2} \mathrm{HPO}_{4} / 0.2 \mathrm{M} \mathrm{NaH}_{2} \mathrm{PO}_{4}, \mathrm{pH} 7.4$ ) and $50 \mu \mathrm{L} \mathrm{D}_{2} \mathrm{O}$. The sample was then centrifuged at $12000 \times \mathrm{g}$ at $4{ }^{\circ} \mathrm{C}$ for $20 \mathrm{~min}$, and the supernatant was transferred into a 5-mm NMR tube.

\section{NMR measurements}

Nuclear magnetic resonance (NMR) spectroscopy experiments were performed on a Bruker Avance III NMR spectrometer equipped with a cryogenic probe at $600.17 \mathrm{MHz}$ for ${ }^{1} \mathrm{H}$ observation at $298 \mathrm{~K}$. Thawed serum $(200 \mu \mathrm{L})$ was buffered with $400 \mu \mathrm{L}$ phosphate buffer $\left(0.2 \mathrm{M} \mathrm{Na}_{2} \mathrm{HPO}_{4} / 0.2 \mathrm{M} \mathrm{NaH}_{2} \mathrm{PO}_{4}, \mathrm{pH}\right.$ 7.4) and $50 \mu \mathrm{L} \mathrm{D}_{2} \mathrm{O}$ prior to its transfer into a $5-\mathrm{mm}$ NMR tube. Transverse relaxation-edited spectra were recorded using the watersuppressed Carr-Purcell-Meiboom-Gill (CPMG) pulse sequence [RD- $\left.90^{\circ}-\left(\tau-180^{\circ}-\tau\right)_{n}-A C Q\right]$. A fixed total spin-spin relaxation delay of $2 \mathrm{nt}$ for $120 \mathrm{~ms}$ was applied to attenuate the broad NMR signals of slowly tumbling molecules with short $\mathrm{T}_{2}$ relaxation times and to retain signals of low-molecular weight compounds. Sixty-four transients were collected into $32 \mathrm{~K}$ data points with a spectral width of $20 \mathrm{ppm}$ and an acquisition time of $2.73 \mathrm{~s}$.

NMR data for each pancreatic tissue sample were obtained using a solvent-suppressed $1 \mathrm{D}{ }^{1} \mathrm{H}$ ZGPR pulse sequence (RD-90 ${ }^{\circ}$ ACQ). The ${ }^{1} \mathrm{H}$ ZGPR spectra were recorded with four dummy scans and 128 transients into $32 \mathrm{~K}$ data points with a spectral width of $20 \mathrm{ppm}$, a relaxation delay of $10.0 \mathrm{~s}$ and an acquisition time of $2.73 \mathrm{~s}$. All 1D spectra were processed with an exponential function with a $0.03 \mathrm{~Hz}$ line broadening factor and zero-filling to $64 \mathrm{~K}$ data points. Moreover, 2D pulsed field gradient correlation spectroscopy (gCOSY), together with 2D homonuclear total correlation spectroscopy (TOCSY) of selected serum and pancreas tissue samples were performed with standard Bruker pulse programs for the confirmation of chemical shift assignments.

\section{Multivariate statistical analysis}

A total of 76 spectra corresponding to serum and pancreas samples were considered for multivariate and univariate analysis. The preprocessing protocol of all $1 \mathrm{D}^{1} \mathrm{H}$ raw NMR spectra was the same as that described in our previous work [19]. The spectral regions of each metabolite were selected and binned into one integral. The integrals of the resulting 27 metabolites in serum and 33 metabolites in pancreas tissue were normalized to the sum of the spectral intensities (excluding the regions of the residual water and organic solvent resonances) to compensate for differences in the concentrations of samples. Subsequently, the integral values were submitted to unit variance (UV) scaling and then subjected to multivariate analysis by principal component analysis (PCA) and orthogonal partial least squares projection to latent structures modeling-discriminant analysis (OPLS-DA) using the SIMCA-P + 14.0 software package (Umetrics, Umeå, Sweden). The PCA score plots were visualized with the first principal component $(t[1])$ and the second principal component (t[2]), while the OPLS-DA plots were visualized with the first principal component $(\mathrm{t}[1])$ and the orthogonal component (to[1]). The parameters Q2 (cum) and R2X (cum) were supplied by the software to assess the quality of the model. R2X (cum) is the total variation explained in the data, and Q2 (cum) is the cross-validated explained variation.

The correlation coefficient $(r)$ for assessing the relationship between the variations and the first component of OPLS-DA models and the variable importance in the projection (VIP) values (threshold >1) was extracted to identify the metabolites with 


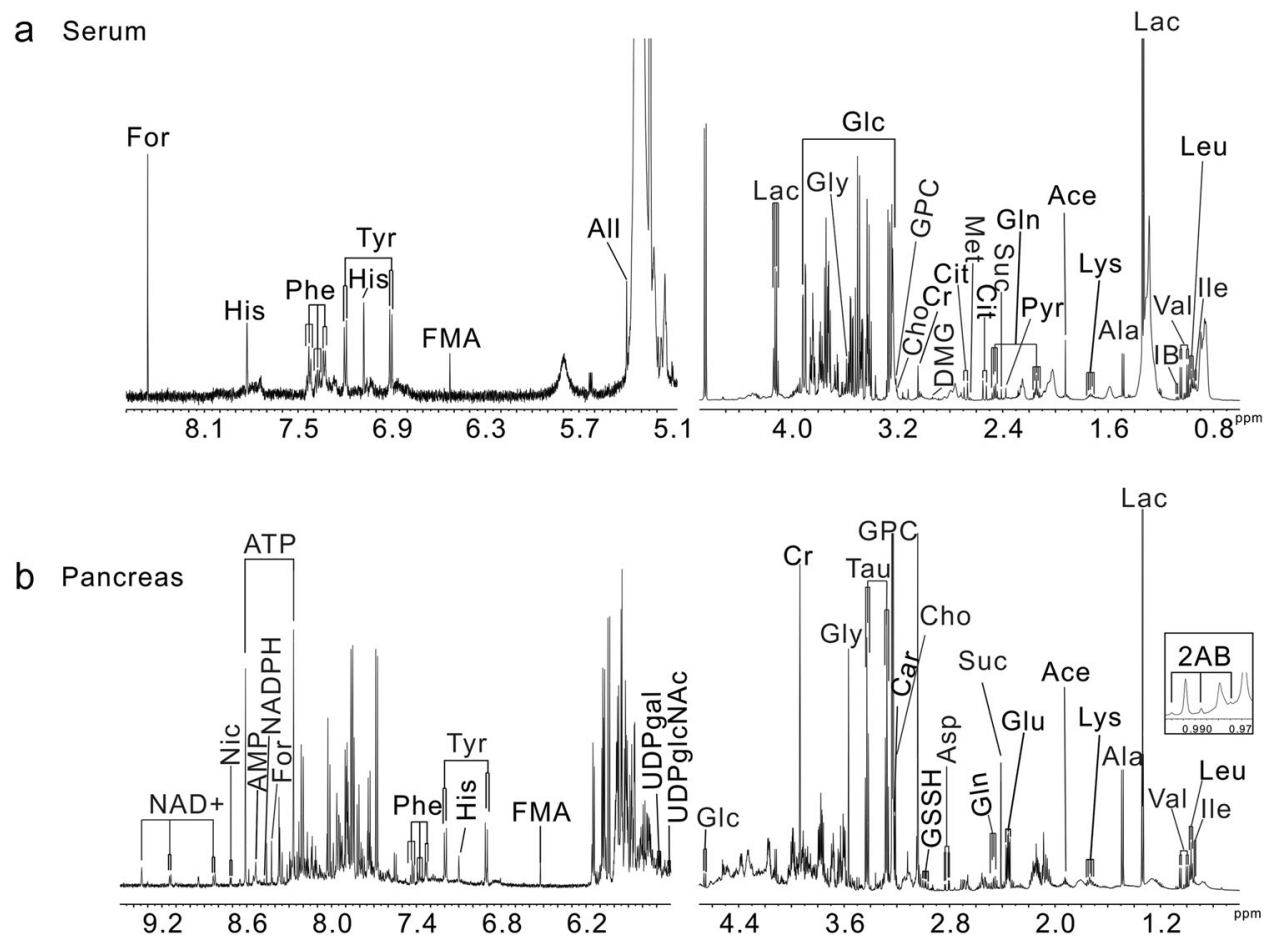

Fig. 1 The representative ${ }^{1} \mathrm{H}$ NMR CPMG spectrum $(\delta 0.6-4.7,5.0-8.6)$ of the serum sample from the APP/PS1 group mouse and the representative $600 \mathrm{MHz}{ }^{1} \mathrm{H}$ NMR ZGPR spectrum ( $\left.\delta 0.6-4.7,5.6-9.6\right)$ of aqueous extract from the pancreatic tissue of the APP/PS1 group mouse. The abbreviations of metabolites are shown in Table S1

significant contributions to the separations between studied groups. Additionally, the relative changes of metabolites between groups were calculated using the normalized integrals, i.e., $\left(I_{\mathrm{A}}-I_{\mathrm{B}}\right) / I_{\mathrm{B}}$, in which $I_{\mathrm{A}}$ and $I_{\mathrm{B}}$ represent the mean metabolite integrals corresponding to group $A$ and group $B$ for comparison in one analysis model. The relative integrals of metabolites between groups are also illustrated by heatmaps.

Univariate statistics of metabolite integrals

The group means of metabolite integrals are expressed as the mean \pm SEM. Significant differences in intergroup variation were evaluated by a nonparametric Wilcoxon test using SPSS 17.0. Statistical significance was considered at $P<0.05$. Box plots of each metabolite with statistical significance were also illustrated to present the group integral differences by Graph Pad Prism version 5.01 software (Graph Pad Software Inc., San Diego, CA, USA).

System statistical metabolic correlation analysis and hierarchical cluster analysis

To explore latent relationships among metabolites and the interrupted metabolic pathways, we carried out Pearson's correlation coefficient calculation and hierarchical cluster analysis based on the relative integrals of metabolites between groups.

\section{RESULTS}

Metabolites identified in the ${ }^{1} \mathrm{H}$ NMR spectra of serum and pancreatic tissue

The ${ }^{1} \mathrm{H}$ NMR spectra presented in Fig. 1 provide comprehensive snapshots of metabolites from the serum and aqueous extracts of pancreatic tissues, which are dominated by organic acids (isobutyrate, lactate, citrate, pyruvate, succinate, fumarate, 2-aminobutyrate, acetate, formate, taurine, 2-aminobutyrate), amino acids (leucine, isoleucine, valine, alanine, lysine, glycine, tyrosine, methionine, phenylalanine aspartate, glutamine, glutamate, histidine), energy metabolites (ATP, AMP), carbohydrates (glucose, UDPgal, UDPglcNAC), and others (creatine, Glc1P, NAD ${ }^{+}$, nicotinurate, NADPH, choline, GPC, carnitine, GSSH, allantoin, dimethylglycine). The assignments (both chemical shift and multiplicity) of identified metabolites (Supplementary Table S1) were obtained by referring to the previously published literature and confirmed by $2 \mathrm{D}^{1} \mathrm{H}-{ }^{1} \mathrm{H}$ COSY and TOCSY spectra.

Multivariate analysis of ${ }^{1} \mathrm{H}$ NMR data for serum samples from experimental mice

The metabolic fingerprints composed of the integrals of metabolites were analyzed by PCA to evaluate the unbiased clustering and unsupervised separations among the APP and WT groups. The PCA scatter plots (Supplementary Fig. S1) derived from the NMR data of serum samples of the experimental mice present clear separations by gender in the first principal component and clustering tendencies for APP9m mice vs. WT9m mice in the second principal component. To determine the discriminative metabolites contributing to the separations between the APP group mice and the WT group mice, we established OPLS-DA models (Fig. 2). The satisfactory robustness of the OPLS-DA score plots was demonstrated by values of Q2 larger than 0.4. Both APP6m mice vs. WT6m mice and APP9m mice vs. WT9m mice showed clear separations in the OPLS-DA score plots, indicating that severe global metabolic perturbations were induced by the progression of $A D$. Based on the determined coefficient numbers of $(|r|)$ extracted from the s-line plots, VIP (threshold $>1$ ) from the OPLS-DA coefficient plots, and the $P$ values (threshold $<$ 0.05 ) from the Wilcoxon tests of univariate statistical analysis, the major discriminative metabolites for the compared groups were identified in a pair-wise comparison (Fig. 3, Table 1). Decreased levels of fumarate, pyruvate, glucose-1-phosphate, choline, and taurine were observed in the serum samples of APP6m group mice (Fig. 3a). In addition, the levels of glucose-1-phosphate, choline, and taurine were positively correlated with each other, and the level of pyruvate was positively correlated with the level of glucose-1-phosphate in the experimental mice at the age of 6 month old (Fig. 3b). Compared with the corresponding WT9m group, the APP9m group exhibited 

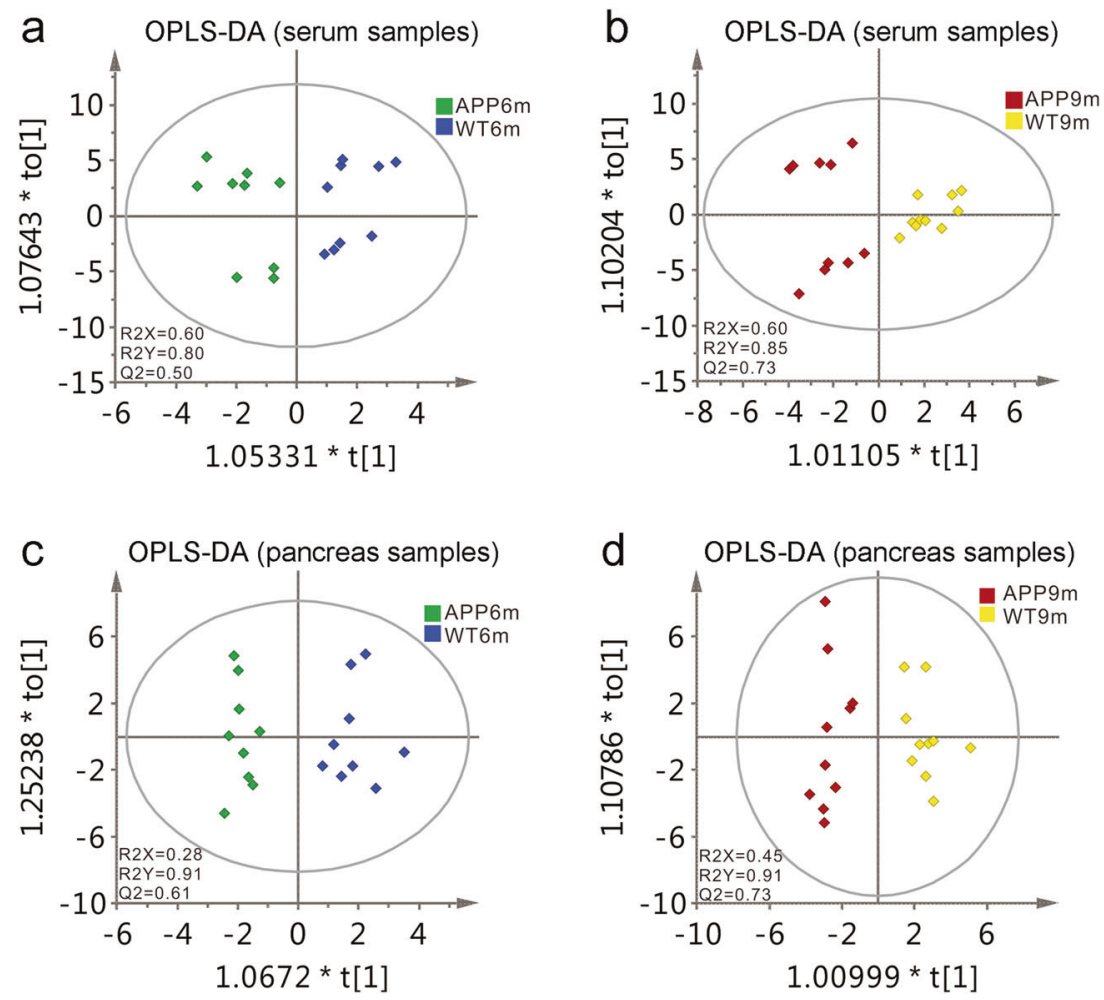

Fig. 2 The OPLS-DA score plots derived from the $600 \mathrm{MHz}{ }^{1} \mathrm{H}$ NMR spectra of serum samples (a, b) and pancreas samples (c, $\left.\mathbf{d}\right)$ extracted from the mice in control groups (WT6m, WT9m) and APP/PS1 transgenic groups (APP6m, APP9m). Each group contains 9 or 10 samples $(n=9$ or $n=10$ ). The values of Q2 parameter, which were greater than 0.4 , indicated that the established OPLS-DA models were valid

Table 1. Quantitative comparisons of metabolites in serum of experimental mice

\begin{tabular}{lll}
\hline Metabolites in serum & $\begin{array}{l}\text { APP6m vs WT6m } \\
\text { (model vs control) } \\
\text { \%Average changes } \\
(|r|, \text { VIP, P-Value) } \\
(|r|>=0.47)\end{array}$ & $\begin{array}{l}\text { APP9m vs WT9m } \\
\text { (model vs control) } \\
\text { \%Average changes } \\
(|r|, \text { VIP, } P \text {-Value) } \\
(|r|>=0.44)\end{array}$ \\
\hline Gln & - & $15.59(0.65,1.23,0.01)$ \\
Glc & - & $25.90(0.62,1.2,0)$ \\
Glc1p & $-54.89(0.83,1.94,0)$ & - \\
FMA & $-39.80(0.72,1.65,0)$ & - \\
All & - & $22.63(0.68,1.27,0.01)$ \\
Ace & - & $130.17(0.66,1.37,0)$ \\
Ala & - & $37.83(0.62,1.32,0.03)$ \\
Val & - & $33.39(0.66,1.34,0.01)$ \\
Tau & $-30.32(0.6,1.52,0.02)$ & $17.12(0.65,1.22,0.02)$ \\
Ser & - & $25.10(0.72,1.34,0)$ \\
Pyr & $-19.91(0.73,1.65,0.01)$ & $-19.65(0.53,1.08,0.02)$ \\
Leu & - & $40.12(0.69,1.35,0)$ \\
Lac & - & $-8.55(0.65,1.3,0.01)$ \\
Cho & $-28.18(0.72,1.69,0)$ & - \\
\hline
\end{tabular}

Gln glutamine, Glc glucose, Glc1P Glucose 1-phosphate, FMA fumarate, All allantoin, Ace acetate, Ala alanine, Val valine, Tau taurine, Ser serine, Pyr pyruvate, Leu leucine, Lac lactate, Cho choline. "-" means no significant change

decreased levels of lactate and pyruvate and increased levels of glutamine, taurine, allantoin, acetate, alanine, leucine, valine, serine, and glucose in the serum (Fig. 3c). Among the identified discriminative metabolites for the serum samples of APP9m mice vs.
WT9m mice, the levels of leucine, valine, acetate and alanine were positively correlated with each other and negatively correlated to the level of pyruvate (Fig. 3d).

Metabolic disorders observed in the pancreatic tissues of $A D$ mice To investigate the metabolic events that occurred in the pancreatic tissues of the AD mice, we performed a series of multivariate analyses using the integrals of the pancreatic metabolites from the experimental mice in the APP6m vs. WT6m and APP9m vs. WT9m groups. As illustrated by the OPLSDA models, the metabolic profiles of APP group mice were clearly different from those in the WT group mice along $t$ [1] (Fig. 2c, d). The key metabolites that were significantly disturbed by $A D$ progression and the predominant contributors to the group separations of the pancreatic samples were identified accordingly (Figs. 4 and 5b, Table 2). In comparison with the pancreatic tissue of the WT6m group, the pancreatic tissue of the APP $6 \mathrm{~m}$ group exhibited higher levels of 2-aminobutyrate, valine, and alanine (Figs. 4a and 5b, Table 2). Additionally, the pancreatic tissue samples of the APP9m group exhibited higher levels of metabolites NADPH, betaine, ATP, succinate, lactate, glucose, malate, 2-aminobutyrate, citrate, and aspartate and lower levels of metabolites glycine, AMP, and nicotinate than the corresponding samples from the WT group (Figs. $4 c$ and $5 b$, Table 2). In addition, the level of glucose was negatively correlated with the level of glycine and positively correlated with the levels of ATP, lactate, malate, 2-aminobutyrate, and citrate (Fig. 4d).

\section{DISCUSSION}

A growing body of literature from epidemiological studies and research performed using experimental animal models has demonstrated that in AD progression, T2D-like effects such as insulin resistance might be induced, occurring in both the brain 

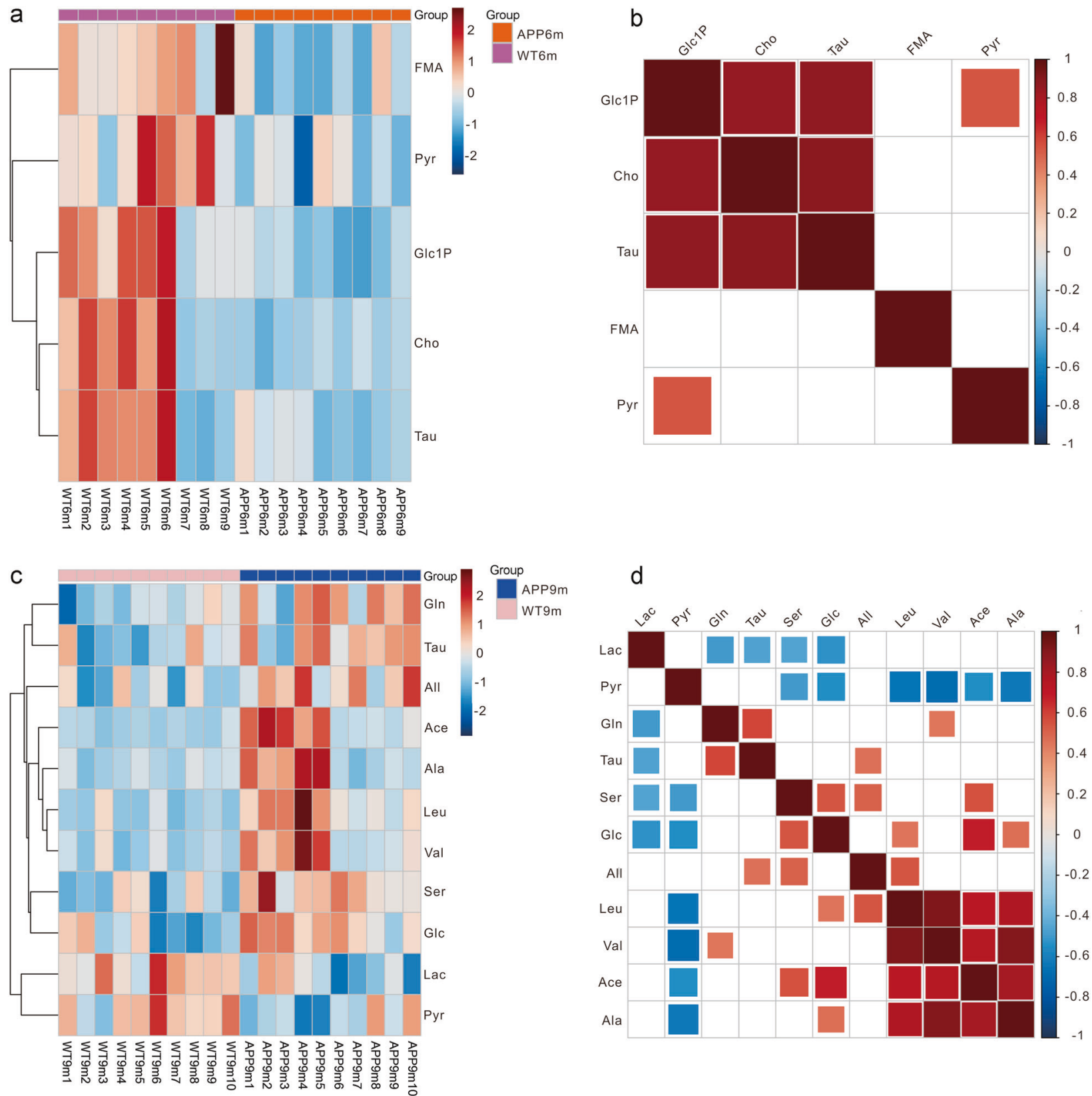

Fig. 3 The heat maps (left panel) and their corresponding correlation plots (right panel) derived from the integral values of metabolites in the serum samples of experimental mice at the age of 6 months (WT6m and APP6m groups, $\mathbf{a}, \mathbf{b}$ ) and of those at the age of 9 months (WT9m and APP9m groups, $\mathbf{c}, \mathbf{d})$

and periphery of $A D$ subjects [20-23]. More interestingly, compared with the observations in the T2D mice (ob/ob mice, high-fat diet-fed mice), more severe glucose metabolism dysfunctions, including glucose intolerance, insulin resistance and insulin signaling impairment, have been detected in $\mathrm{APP}^{+}$-ob/ob mice and high-fat diet-fed APP/PS1 mice [24-26]. All the abovementioned findings indicate that the pathogenesis of both $A D$ and T2D share a common metabolic background. In fact, Jill K. Morris and his colleagues have reported that the metabolomics profiles of T2D patients and AD patients are quite similar to each other [15]. Although $A D$ and T2D have been demonstrated to correlate with each other, more data are still needed to elucidate the underlying molecular mechanisms.

Metabolomics can reveal metabolic profile changes of living systems in response to external stimuli [27-29] and has proven to be valuable in the investigation of molecular mechanisms underlying various human diseases, including Alzheimer's disease [30-36]. In this study, the combined use of nontargeted metabolomics, metabolic correlation analysis, and multiple statistical approaches was applied to detect the metabolic alterations of the serum and pancreatic tissues of APP/PS1 mice at different time points during $A D$ progression. In addition, the metabolic mechanisms of the metabolic correlations between AD and its T2D-promoting effects were then investigated. To the best of our knowledge, this is the first study to characterize the metabolic phenotype of the pancreas throughout the development of $A D$ in a rodent animal model.

As is well known, amyloid plaques formation within the brain caused by the aggregation of $\beta$-amyloid $(A \beta)$ polypeptides is one of the pathological hallmarks of $A D$. Considerable literature has demonstrated that APP/PS1 mice just begin to show occasional $A \beta$ deposits at 6 month of age $[37,38]$, with severe astrocytosis 

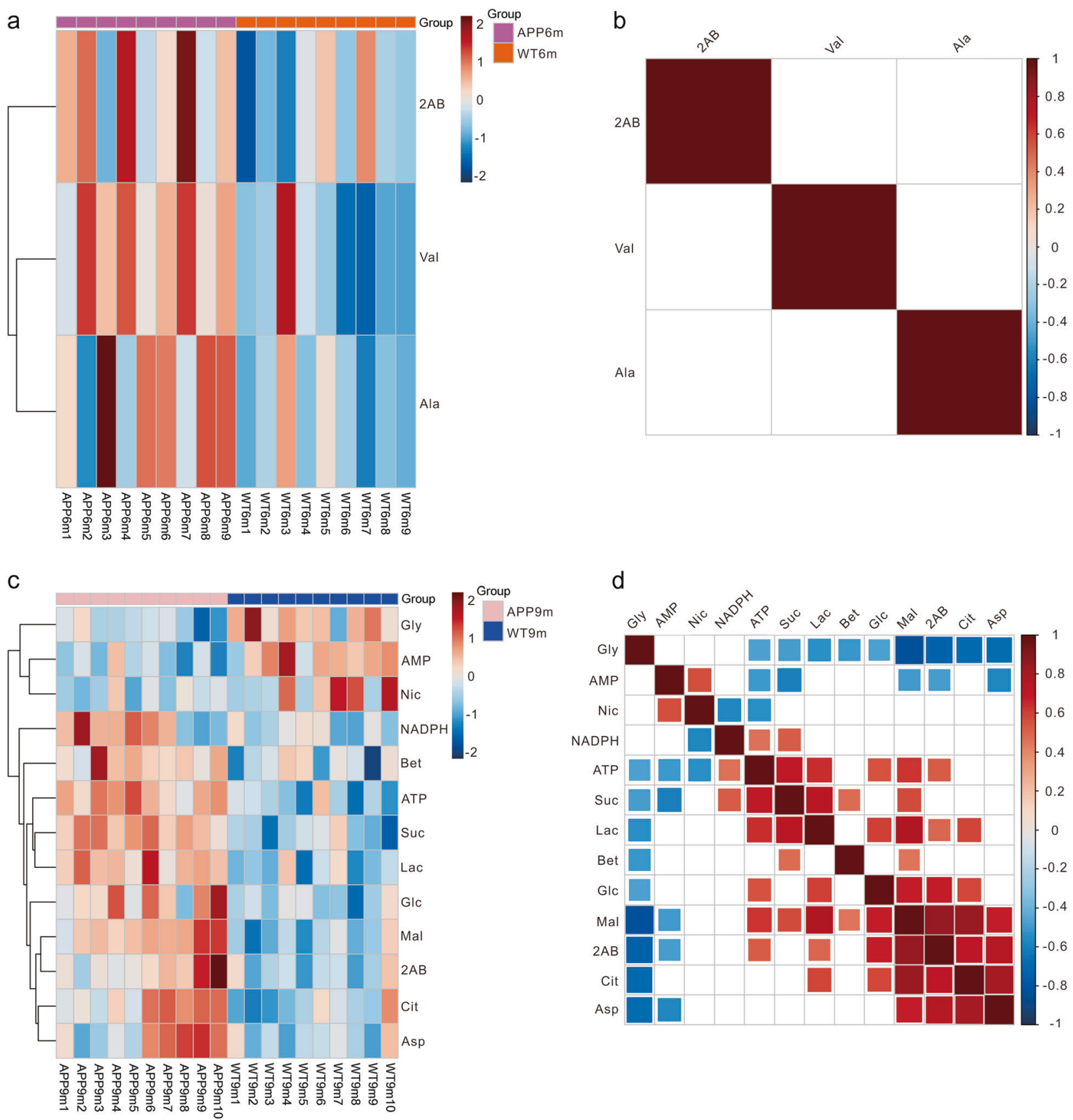

Fig. 4 The heat maps (left panel) and their corresponding correlation plots (right panel) derived from the integral values of metabolites in the pancreatic tissues of experimental mice at the age of 6 month old (WT6m and APP6m groups, a, b) and of those at the age of 9 month old (WT9m and APP9m groups, $\mathbf{c}, \mathbf{d}$ )

developing in parallel with plaque deposition [39]. At the age of 9 month, abundant plaques appear in the hippocampus and cortex of the transgenic mice, and more neuronal loss is observed adjacent to plaques than in more distal areas [40]. Therefore, in this study, 6-month-old and 9-month-old APP/PS1 mice were selected to mimic the early stage and the more developed stage of the $A \beta$ cascade associated with the progression of $A D$. According to previously published works, $A \beta$ plaques might directly induce insulin resistance, insulin signaling dysfunction, and alterations in whole-body metabolism $[13,41]$. In our study, consistent with the global effects of the continuous deposition of $A \beta$ peptides in brain tissues, glucose metabolism disorders were detected globally (serum) and locally (pancreas) in AD mice.
Compared with the wild-type mice at the age of 9 month old, APP9m mice exhibited significant upregulation of the glucose level (hyperglycemia) in both the serum (fold of change $=25.90 \%$, Table 1) and pancreas (fold of change $=20.84 \%$, Table 2), which might contribute to the cognitive decline, aggravated insulin resistance, and pancreatic hyperglycemia damage associated with $\mathrm{AD}$ progression $[42,43]$.

In addition to damages to the central nervous system and peripheral organs regulating glucose metabolism in living systems, hyperglycemia also induces disturbances in the generation and scavenging of reactive oxygen species, disorders of energy metabolism, and upregulation of inflammation [43-45]. In our case, due to the oxidative stress associated with the global 
a

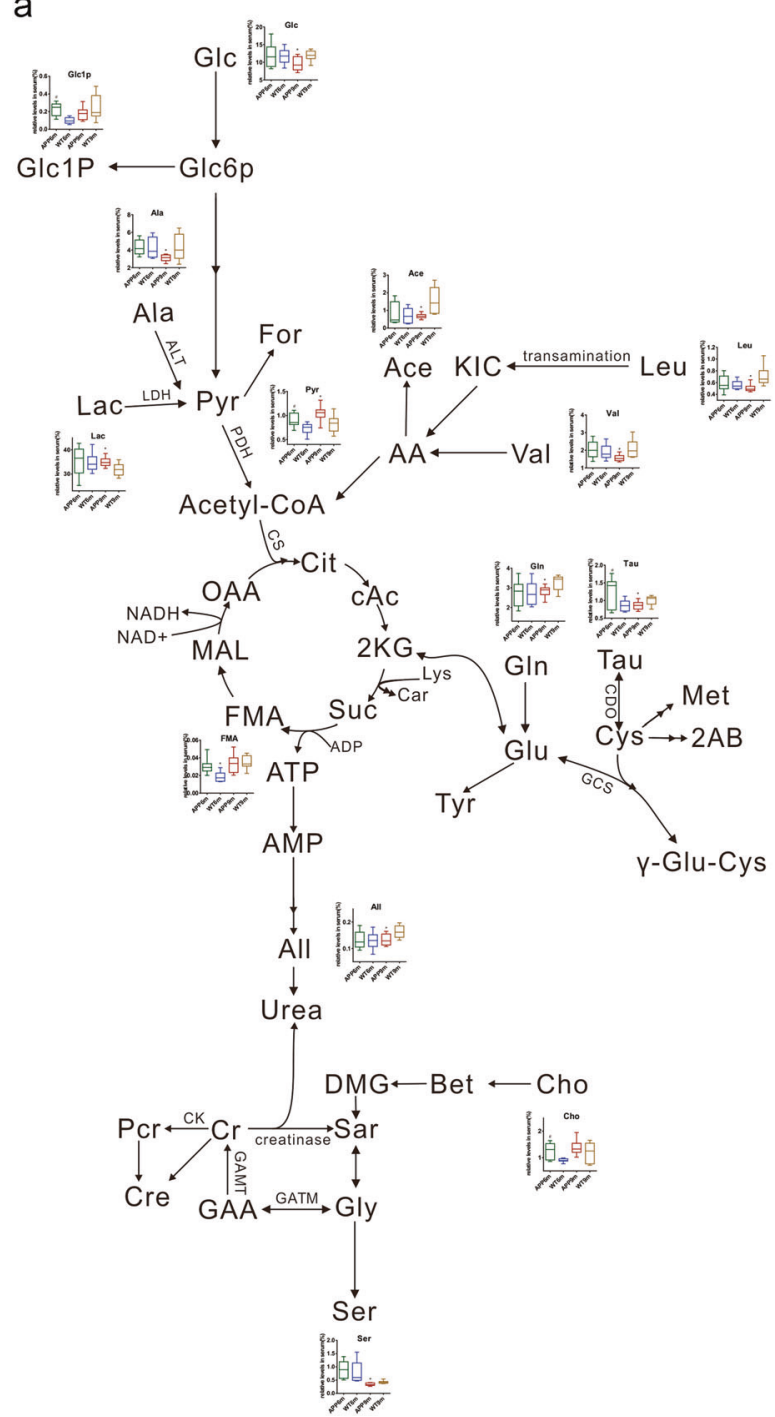

b

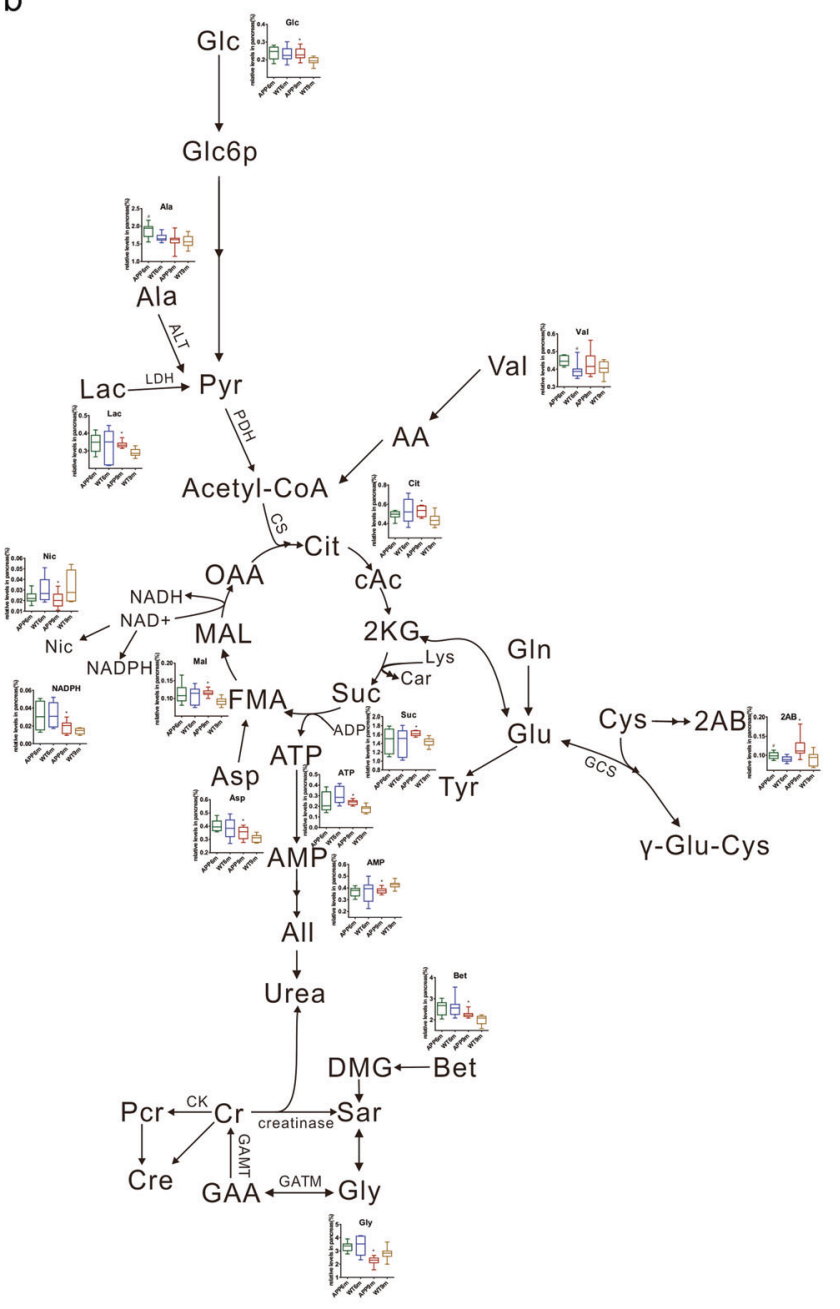

Fig. 5 The potential metabolic pathways disturbed by the progress of $A D$ in the serum (a) and pancreas tissue (b) of the experimental mice. Some abbreviations of metabolites are shown in Table S1. The others are as follows: Glc6P glucose-6-phosphate, AA acetoacetate, OAA oxaloacetate, CAc cis-aconitate, 2KG 2-ketoglutarate, ADP adenosine diphosphate, NADH reduced nicotinamide adenine dinucleotide, Met methionine, Cys cysteine, $\gamma$-Glu-Cys L- $\gamma$-Glutamylcysteine, Pcr creatine phosphate, Cre creatinine, GAA guanidinoacetate, Sar sarcosine, DMG dimethylglycine, Ser serine, CS citrate synthase, ALT alanine transaminase, PDH pyruvate dehydrogenase, LDH lactate dehydrogenase, GCS $\gamma$-glutamylcysteine synthetase, CDO cysteine dioxygenase, GATM glycine amidinotransferase, GAMT guanidinoacetate $\mathrm{N}$-methyltransferase, CK creatine kinase

hyperglycemia of $A D$ mice, decreased levels of taurine (fold of change $=-30.32 \%$, Table 1 ) in the serum of AD mice at the age of 6 month old and increased levels of allantoin (fold of change $=$ $22.63 \%$, Table 1) in the serum of AD mice at the age of 9 month old were observed. Taurine may serve as a protector against oxidative injury by modulating the synthesis of mitochondrial proteins and upregulating the activity of the mitochondrial respiratory chain [46-48]. Allantoin is a product of the oxidation of uric acid via a nonenzymatic pathway with the presence of high levels of reactive oxygen species [49]. In addition to ROS stress, disorders of energy metabolism were also observed in the serum and pancreas of $A D$ mice. One of the key intermediates of the Krebs cycle, fumarate, was significantly downregulated (fold of change $=-39.80 \%$, Table 1 ) in the serum of AD mice at the age of 6 month old. The other Krebs cycle intermediate, pyruvate, was downregulated in the serum of AD mice at the ages of 6 month old (fold of change $=-19.91 \%$, Table 1) and 9 month old (fold of change $=-19.65 \%$, Table 1). Meanwhile, decreased levels of two intermediate metabolites of glycolysis, glucose-1-phosphate (fold of change $=-54.89 \%$, Table 1 ) and lactate (fold of change $=$ $-8.55 \%$, Table 1 ), were detected in the serum of AD mice at the age of 6 month old and 9 month old, respectively. In the metabolite correlation analysis of the serum samples from the experimental mice at the age of 6 month the change in glucose-1phosphate was significantly and positively correlated with the alteration in pyruvate (Fig. 3b), which suggests a synchronized global downregulation of glycolysis and the Krebs cycle of $A D$ mice. Consistent with the observed alterations in global glucose metabolism and energy metabolism under the circumstance of hyperglycemia, the levels of leucine (fold of change $=40.12 \%$, Table 1 ) and valine (fold of change $=33.39 \%$, Table 1 ) branchedchain amino acids, which have been demonstrated to play a role in responding to the downregulation of glucose utilization $[50,51]$, were upregulated in the serum of APP9m group mice (Table 1), and the observed alteration in leucine was positively correlated with the change in glucose (Fig. 3d). Notably, increased 
Table 2. Quantitative comparisons of aqueous metabolites in pancreas tissues of experimental mice

\begin{tabular}{|c|c|c|}
\hline Metabolites in pancreas & $\begin{array}{l}\text { APP6m vs WT6m } \\
\text { (model vs control) } \\
\text { \%Average changes } \\
(|r|, \text { VIP, P-Value) } \\
(|r|>=0.47)\end{array}$ & $\begin{array}{l}\text { APP9m vs WT9m } \\
\text { (model vs control) } \\
\text { \%Average changes } \\
(|r|, \text { VIP, P-Value) } \\
(|r|>=0.44)\end{array}$ \\
\hline Val & $14.31(0.71,1.91,0)$ & - \\
\hline Suc & - & $13.56(0.83,1.64,0)$ \\
\hline $2 A B$ & $10.43(0.47,1.44,0.03)$ & $32.37(0.58,1.38,0.01)$ \\
\hline $\mathrm{Nic}$ & - & $-36.80(0.5,1.04,0.03)$ \\
\hline NADPH & - & $35.41(0.52,1.19,0.04)$ \\
\hline Mal & - & $27.65(0.8,1.64,0)$ \\
\hline Lac & - & $15.36(0.78,1.54,0)$ \\
\hline Gly & - & $-20.27(0.66,1.39,0)$ \\
\hline Glc & - & $20.84(0.69,1.41,0)$ \\
\hline Cit & - & $21.14(0.58,1.27,0)$ \\
\hline Bet & - & $11.75(0.56,1.11,0.01)$ \\
\hline ATP & - & $36.50(0.86,1.7,0)$ \\
\hline Asp & - & $13.55(0.44,1.14,0.03)$ \\
\hline AMP & - & $-11.46(0.65,1.31,0)$ \\
\hline Ala & $11.58(0.56,1.57,0.02)$ & - \\
\hline
\end{tabular}

Val valine, Suc succinate, 2-AB 2-aminobutyrate, Nic nicotinurate, $N A D P H$ reduced nicotinamide adenine dinucleotide phosphate, Mal malate, LaC lactate, Gly glycine, Glc glucose, Cit citrate, Bet betaine, ATP adenosinetriphosphate, Asp aspartate, AMP adenosine-monophosphate, Ala alanine. "-" means no significant change

levels of branched-chain amino acids (BCAAs) in serum have been reported as one of the signatures for the progression of insulin resistance and the onset of T2D [52, 53].

All of the aforementioned global metabolic changes in AD mice strongly suggest that the progression of $A D$ in transgenic mice might promote the development of diabetes. Not surprisingly, metabolic disturbances in energy metabolism were observed in the pancreas of $A D$ mice. Compared with the pancreas of WT mice, the pancreas of AD mice exhibited a higher rate of ATP synthesis and faster cell respiration. Downregulated AMP (fold of change $=-11.46 \%$, Table 2), upregulated ATP (fold of change $=$ $36.50 \%$, Table 2), upregulated glucose (fold of change $=20.84 \%$, Table 2), upregulated lactate (fold of change $=15.36 \%$, Table 2 ), upregulated citrate (fold of change $=21.14 \%$, Table 2), upregulated succinate (fold of change $=13.56 \%$, Table 2 ), and upregulated malate (fold of change $=27.65 \%$, Table 2 ) were observed in the pancreatic tissues of the APP9m mice. The change in glucose in the pancreatic tissues of APP9m mice was positively correlated with the alterations in ATP, malate, lactate, and citrate (Fig. 4d), which suggests that the overloaded glucose exerts a dramatic upregulation of energy metabolism. In addition, the upregulation of ATP might at least be partially attributed to the enhanced need for insulin biosynthesis under the circumstance of global hyperglycemia. Insulin biosynthesis is an ATP-dependent process [54]. During AD progression, metabolic disturbances in the intermediate metabolites of the oxidative respiratory chain were also observed. Lower levels of nicotinurate and higher levels of NADPH were detected in the pancreas of APP9m group mice than in that of WT9m group mice (Table 2). Nicotinurate is the major detoxification product of nicotinic acid, the derivatives of which, such as NADH, NAD, NAD ${ }^{+}$, and NADP, play essential roles in the energy metabolism of living cells. More importantly, the disturbed glucose metabolism in KK-Ay obese mice has been shown to be improved by nicotinic acid treatment, which functions by suppressing the effect of the increase in the blood glucose level [55]. Therefore, downregulation of nicotinurate in the pancreas of $A D$ mice might be a consequence of the reduced insulin sensitivity and the development of insulin resistance.

Finally, we should mention that gender-dependent separations in the metabolomics profiles of the experimental mice were observed in this study. The PCA scatter plots (Supplementary Fig. S1) derived from the NMR data of the serum and pancreatic samples of the experimental mice at the age of 6 months present separations by gender in the first principal component. Meanwhile, clustering tendencies are observed for APP9m mice vs. WT9m mice at the age of 9 month in the second principal component (Supplementary Fig. S1). Therefore, we conclude that the global glucose metabolism changes induced by the development of Alzheimer's disease might be gender dependent, at least for the APP/PS1 transgenic mice at the age of 6 month old. A further metabolomics study incorporating more experimental mice might provide a more thorough picture of the gender-based metabolomics features linked with $A D$ progression.

\section{CONCLUSION}

In summary, the metabolomics data presented in this manuscript suggest a tight correlation between $A D$ progression and disorders of global glucose metabolism. Hyperglycemia, its accompanying metabolic disorders, including oxidative stress and energy metabolism imbalance, and the upregulation of branched-chain amino acids were observed in the serum of AD mice (Fig. 5a). In addition, global glucose metabolism dysfunctions, particularly hyperglycemia, also induced a metabolic profile change in the pancreas of AD mice. Moreover, the overloaded glucose caused a dramatic upregulation of energy metabolism in the pancreas of $A D$ mice (Fig. 5b). The aforementioned metabolic changes in the serum and pancreas of $A D$ mice indicate that the development of $A D$ promotes diabetes by downregulating the global utilization of glucose and interfering with the function of the pancreas. Our findings reveal a tight correlation between the pathogenesis of $A D$ and glucose metabolism dysfunctions, indicating that targeting the glucose metabolism-related metabolic manifestations of $A D$ might be an effective strategy to treat this currently uncurable disease.

\section{ACKNOWLEDGEMENTS}

This work was financially supported by the National Natural Science Foundation of China (Grant no. 21778061) and the National Science \& Technology Major Project "Key New Drug Creation and Manufacturing Program" of China (Grant no. 2018ZX09711002)

\section{AUTHOR CONTRIBUTIONS}

$\mathrm{XL}, \mathrm{WW}, \mathrm{HYZ}$ and $\mathrm{NXZ}$ designed the study, $\mathrm{XL}, \mathrm{HYZ}$ and $\mathrm{NXZ}$ wrote the manuscript, and $\mathrm{XL}, \mathrm{WW}$ and $\mathrm{HLC}$ performed the experiments.

\section{ADDITIONAL INFORMATION}

The online version of this article (https://doi.org/10.1038/s41401-019-0239-3) contains supplementary material, which is available to authorized users.

Conflict of interest: The authors declare that they have no conflict of interest.

\section{REFERENCES}

1. Kim B, Feldman EL. Insulin resistance as a key link for the increased risk of cognitive impairment in the metabolic syndrome. Exp Mol Med. 2015;47:e149.

2. Neth BJ, Craft S. Insulin resistance and Alzheimer's disease: bioenergetic linkages. Front Aging Neurosci. 2017;9:345. 
3. Waither G, Obert P, Dutheil F, Chapier R, Lesourd B, Naughton G, et al. Metabolic syndrome individuals with and without type 2 diabetes mellitus present generalized vascular dysfunction cross-sectional study. Arter Throm Vas. 2015;35:1022-U318.

4. Blazquez E, Velazquez E, Hurtado-Carneiro V, Ruiz-Albusac JM. Insulin in the brain: its pathophysiological implications for states related with central insulin resistance, type 2 diabetes and Alzheimer's disease. Front Endocrinol. 2014;5:161.

5. Kuljis RO, Salkovic-Petrisic M. Dementia, diabetes, Alzheimer's disease, and insulin resistance in the brain: progress, dilemmas, new opportunities, and a hypothesis to tackle intersecting epidemics. J Alzheimers Dis. 2011;25:29-41.

6. de Nazareth AM. Type 2 diabetes mellitus in the pathophysiology of Alzheimer's disease. Dement Neuropsychol. 2017;11:105-13.

7. Wegiel J, Wisniewski HM, Muzylak M, Tarnawski M, Badmajew E, Nowakowski J, et al. Fibrillar amyloid-beta production, accumulation, and recycling in transgenic mice pancreatic acinar cells and macrophages. Amyloid. 2000;7:95-104.

8. Figueroa DJ, Shi XP, Gardell SJ, Austin CP. Abetapp secretases are co-expressed with Abetapp in the pancreatic islets. J Alzheimers Dis. 2001;3:393-96.

9. Lu Z, Xie J, Yan R, Yu Z, Sun Z, Yu F, et al. A pilot study of pancreatic islet amyloid PET imaging with $\left[{ }^{18}\right.$ F]FDDNP. Nucl Med Commun. 2018;39:659-64.

10. Kurochkin IV, Guarnera E, Berezovsky IN. Insulin-degrading enzyme in the fight against Alzheimer's disease. Trends Pharm Sci. 2018;39:49-58.

11. Kimura N. Diabetes mellitus induces Alzheimer's disease pathology: histopathological evidence from animal models. Int J Mol Sci. 2016;17:503.

12. Bharadwaj $P$, Wijesekara $N$, Liyanapathirana $M$, Newsholme $P$, Ittner $L$, Fraser $P$, et al. The link between type 2 diabetes and neurodegeneration: roles for amyloidbeta, amylin, and tau proteins. J Alzheimers Dis. 2017;59:421-32.

13. Ahmed S, Mahmood Z, Zahid S. Linking insulin with Alzheimer's disease: emergence as type III diabetes. Neurol Sci. 2015;36:1763-69.

14. Bosco D, Fava A, Plastino M, Montalcini T, Pujia A. Possible implications of insulin resistance and glucose metabolism in Alzheimer's disease pathogenesis. J Cell Mol Med. 2011;15:1807-21.

15. Morris JK, Piccolo BD, Shankar K, Thyfault JP, Adams SH. The serum metabolomics signature of type 2 diabetes is obscured in Alzheimer's disease. Am J Physiol Endocrinol Metab. 2018;314:E584-96.

16. Zhong F, Liu X, Zhou Q, Hao X, Lu Y, Guo S, et al. ' ${ }^{1} \mathrm{H}$ NMR spectroscopy analysis of metabolites in the kidneys provides new insight into pathophysiological mechanisms: applications for treatment with Cordyceps sinensis. Nephrol Dial Transpl. 2012;27:556-65.

17. Liu X, Zhong F, Tang XL, Lian FL, Zhou Q, Guo SM, et al. Cordyceps sinensis protects against liver and heart injuries in a rat model of chronic kidney disease: a metabolomic analysis. Acta Pharm Sin. 2014;35:697-706.

18. Zhang T, Wang W, Huang J, Liu X, Zhang H, Zhang N. Metabolomic investigation of regional brain tissue dysfunctions induced by global cerebral ischemia. BMC Neurosci. 2016;17:25

19. Ma HF, Liu X, Wu Y, Zhang NX. The intervention effects of acupuncture on fatigue induced by exhaustive physical exercises: a metabolomics investigation. Evid Based Complement Altern Med. 2015;2015:508302.

20. Jolivalt CG, Lee CA, Beiswenger KK, Smith JL, Orlov M, Torrance MA, et al Defective insulin signaling pathway and increased glycogen synthase kinase-3 activity in the brain of diabetic mice: parallels with Alzheimer's disease and correction by insulin. J Neurosci Res. 2008;86:3265-74.

21. Janson J, Laedtke T, Parisi JE, O'Brien P, Petersen RC, Butler PC. Increased risk of type 2 diabetes in Alzheimer disease. Diabetes. 2004;53:474-81.

22. Steen E, Terry BM, Rivera EJ, Cannon JL, Neely TR, Tavares R, et al. Impaired insulin and insulin-like growth factor expression and signaling mechanisms in Alzheimer's disease - is this type 3 diabetes? J Alzheimers Dis. 2005;7:63-80.

23. Talbot K, Wang HY, Kazi H, Han LY, Bakshi KP, Stucky A, et al. Demonstrated brain insulin resistance in Alzheimer's disease patients is associated with IGF-1 resistance, IRS-1 dysregulation, and cognitive decline. J Clin Invest. 2012;122:1316-38.

24. Takeda S, Sato N, Uchio-Yamada K, Sawada K, Kunieda T, Takeuchi D, et al. Diabetes-accelerated memory dysfunction via cerebrovascular inflammation and A beta deposition in an Alzheimer mouse model with diabetes. Proc Natl Acad Sci USA. 2010;107:7036-41.

25. Jimenez-Palomares $M$, Ramos-Rodriguez JJ, Lopez-Acosta JF, Pacheco-Herrero $M$, Lechuga-Sancho AM, Perdomo G, et al. Increased A beta production prompts the onset of glucose intolerance and insulin resistance. Am J Physiol Endocrinol Metab. 2012;302:E1373-80.

26. Wijesekara N, Ahrens R, Sabale M, Wu L, Ha K, Verdile G, et al. Amyloid-beta and islet amyloid pathologies link Alzheimer's disease and type 2 diabetes in a transgenic model. FASEB J. 2017;31:5409-18.

27. Gebregiworgis T, Powers R. Application of NMR metabolomics to search for human disease biomarkers. Comb Chem High T Scr. 2012;15:595-610.

28. Zhang AH, Sun H, Qiu S, Wang XJ. NMR-based metabolomics coupled with pattern recognition methods in biomarker discovery and disease diagnosis. Magn Reson Chem. 2013;51:549-56.
29. Dudka I, Kossowska B, Senhadri H, Latajka R, Hajek J, Andrzejak R, et al. Metabonomic analysis of serum of workers occupationally exposed to arsenic, cadmium and lead for biomarker research: a preliminary study. Environ Int. 2014;68:71-81.

30. Koivisto H, Leinonen H, Puurula M, Hafez HS, Barrera GA, Stridh MH, et al. Chronic pyruvate supplementation increases exploratory activity and brain energy reserves in young and middle-aged mice. Front Aging Neurosci. 2016;8:41.

31. Han B, Wang JH, Geng Y, Shen L, Wang HL, Wang YY, et al. Chronic stress contributes to cognitive dysfunction and hippocampal metabolic abnormalities in APP/PS1 mice. Cell Physiol Biochem. 2017;41:1766-76.

32. Zhou Q, Zheng H, Chen JX, Li C, Du Y, Xia HH, et al. Metabolic fate of glucose in the brain of APP/PS1 transgenic mice at 10 months of age: a C-13 NMR metabolomic study. Metab Brain Dis. 2018;33:1661-68.

33. Podlesniy P, Figueiro-Silva J, Llado A, Antonell A, Sanchez-Valle R, Alcolea D, et al. Low cerebrospinal fluid concentration of mitochondrial DNA in preclinical Alzheimer disease. Ann Neurol. 2013;74:655-68.

34. Gonzalez-Dominguez R, Garcia-Barrera T, Vitorica J, Gomez-Ariza JL. Application of metabolomics based on direct mass spectrometry analysis for the elucidation of altered metabolic pathways in serum from the APP/PS1 transgenic model of Alzheimer's disease. J Pharm Biomed. 2015;107:378-85.

35. Gao HL, Zhang AH, Yu JB, Sun $H$, Kong L, Wang $X Q$, et al. High-throughput lipidomics characterize key lipid molecules as potential therapeutic targets of Kaixinsan protects against Alzheimer's disease in APP/PS1 transgenic mice. Chromatogr B. 2018;1092:286-95.

36. Yang $X$, Chen DL, Yang J, Liu T, Hu GY, Liang HL, et al. Effects of oligosaccharides from morinda officinalis on gut microbiota and metabolome of APP/PS1 transgenic mice. Front Neurol. 2018;9:412.

37. Woodhouse A, Fernandez-Martos CM, Atkinson RAK, Hanson KA, Collins JM, O'Mara AR, et al. Repeat propofol anesthesia does not exacerbate plaque deposition or synapse loss in APP/PS1 Alzheimer's disease mice. BMC Anesth. 2018;18:47.

38. Jankowsky JL, Fadale DJ, Anderson J, Xu GM, Gonzales V, Jenkins NA, et al. Mutant presenilins specifically elevate the levels of the 42 residue beta-amyloid peptide in vivo: evidence for augmentation of a 42 -specific gamma secretase. Hum Mol Genet. 2004;13:159-70.

39. Kamphuis W, Mamber C, Moeton M, Kooijman L, Sluijs JA, Jansen AHP, et al. GFAP isoforms in adult mouse brain with a focus on neurogenic astrocytes and reactive astrogliosis in mouse models of Alzheimer disease. PLoS ONE. 2012;7:e42823.

40. Jackson RJ, Rudinskiy N, Herrmann AG, Croft S, Kim JM, Petrova V, et al. Human tau increases amyloid beta plaque size but not amyloid beta-mediated synapse loss in a novel mouse model of Alzheimer's disease. Eur J Neurosci. 2016;44:3056-66.

41. Lee YH, Hsu HC, Kao PC, Shiao YJ, Yeh SHH, Shie FS, et al. Augmented insulin and leptin resistance of high fat diet-fed APPswe/PS1dE9 transgenic mice exacerbate obesity and glycemic dysregulation. Int J Mol Sci. 2018;19:2333.

42. Leahy $\mathrm{JL}$, Bonnerweir S, Weir GC. Beta-cell dysfunction induced by chronic hyperglycemia - current ideas on mechanism of impaired glucose-induced insulin-secretion. Diabetes Care. 1992;15:442-55.

43. Daulatzai MA. Cerebral hypoperfusion and glucose hypometabolism: key pathophysiological modulators promote neurodegeneration, cognitive impairment, and Alzheimer's disease. J Neurosci Res. 2017;95:943-72.

44. Ferreira IL, Resende R, Ferreiro E, Rego AC, Pereira CF. Multiple defects in energy metabolism in Alzheimer's disease. Curr Drug Targets. 2010;11:1193-206.

45. Mosconi L, Berti V, Guyara-Quinn C, McHugh P, Petrongolo G, Osorio RS, et al. Perimenopause and emergence of an Alzheimer's bioenergetic phenotype in brain and periphery. PLoS ONE. 2017;12:e0185926.

46. Das J, Ghosh J, Manna P, Sil PC. Taurine protects acetaminophen-induced oxidative damage in mice kidney through APAP urinary excretion and CYP2E1 inactivation. Toxicology. 2010;269:24-34.

47. Das J, Ghosh J, Manna P, Sil PC. Taurine suppresses doxorubicin-triggered oxidative stress and cardiac apoptosis in rat via up-regulation of $\mathrm{PI3}-\mathrm{K} / \mathrm{Akt}$ and inhibition of p53, p38-JNK. Biochem Pharm. 2011;81:891-909.

48. Chang $C Y$, Shen $C Y$, Kang $C K$, Sher YP, Sheu WHH, Chang CC, et al. Taurine protects HK-2 cells from oxidized LDL-induced cytotoxicity via the ROS mediated mitochondrial and p53-related apoptotic pathways. Toxicol Appl Pharm. 2014;279:351-63.

49. Yardim-Akaydin S, Sepici A, Ozkan Y, Simsek B, Sepici V. Evaluation of allantoin levels as a new marker of oxidative stress in Behcet's disease. Scand J Rheuma. 2006;35:61-64

50. Li T, Zhang Z, Kolwicz SC, Abell L, Roe ND, Kim M, et al. Defective branched-chain amino acid catabolism disrupts glucose metabolism and sensitizes the heart to ischemia-reperfusion injury. Cell Metab. 2017;25:374-85.

51. Newgard CB, An J, Bain JR, Muehlbauer MJ, Stevens RD, Lien LF, et al. A branchedchain amino acid-related metabolic signature that differentiates obese and lean humans and contributes to insulin resistance. Cell Metab. 2009:9:311-26. 
Interplay between Alzheimer's disease and global glucose metabolism $X$ Liu et al.

52. Ruiz HH, Chi T, Shin AC, Lindtner C, Hsieh W, Ehrlich M, et al. Increased susceptibility to metabolic dysregulation in a mouse model of Alzheimer's disease is associated with impaired hypothalamic insulin signaling and elevated BCAA levels. Alzheimers Dement. 2016;12:851-61.

53. Knight EM, Ruiz HH, Kim SH, Harte JC, Hsieh W, Glabe C, et al. Unexpected partial correction of metabolic and behavioral phenotypes of Alzheimer's APP/PSEN1 mice by gene targeting of diabetes/Alzheimer's-related Sorcs1. Acta Neuropathol Commun. 2016;4:16.

54. Wijesekara N, Goncalves RA, De Felice FG, Fraser PE. Impaired peripheral glucose homeostasis and Alzheimer's disease. Neuropharmacology. 2018;136:172-81.

55. Yoshinari O, Igarashi K. Anti-diabetic effect of trigonelline and nicotinic acid, on KK-A(y) mice. Curr Med Chem. 2010;17:2196-202. 